\title{
The Evaluation of Bus Lines
}

\section{Lin Zhang(张琳)}

\section{School of North China Electric Power University(Baoding)}

$$
\text { 1391056377@qq.com }
$$

\begin{abstract}
This paper analyzes and evaluates the efficiency of bus lines from the aspects of line layout, hardware facilities, passenger's comfort, convenience and vehicle's speed. We use DEA evaluation method based on AHP constraint, through multi-objective decision model, to decompose the complex issues into parts.
\end{abstract}

Key words: DEA evaluation method; Bus line; Mathematical model

\section{Introduction}

This paper selects DEA evaluation method based on AHP constraint to evaluate the bus lines, in order to find the shortcomings of the bus line in the construction and operation through a comprehensive analysis and evaluation, to provide decisive basis for the optimization of bus lines.

\section{The establishment of the model}

Through analytic hierarchy process, comparing the importance of the evaluation factors to determine the weight and constructing a judgment matrix, Firstly, matrix is normalized by column;then the regularized elements are added by row;finally, the sum of each row is normalized

\subsection{Calculated value of the weight of the indicators}

\begin{tabular}{|c|c|c|c|}
\hline Category & Target layer & Indicator layer & Weight \\
\hline $\begin{array}{l}\text { input } \\
\text { indicators (v) }\end{array}$ & $\begin{array}{c}\text { The structure of } \\
\text { lines(X1) }\end{array}$ & $\begin{array}{c}\text { The length of line } \\
\text { x(11) } \\
\text { Non-linear } \\
\text { coefficient x(12) } \\
\text { Average distance }\end{array}$ & $\begin{array}{l}0.69 \\
0.23 \\
0.08\end{array}$ \\
\hline
\end{tabular}


between stations

$\mathrm{x}(13)$

The probability that

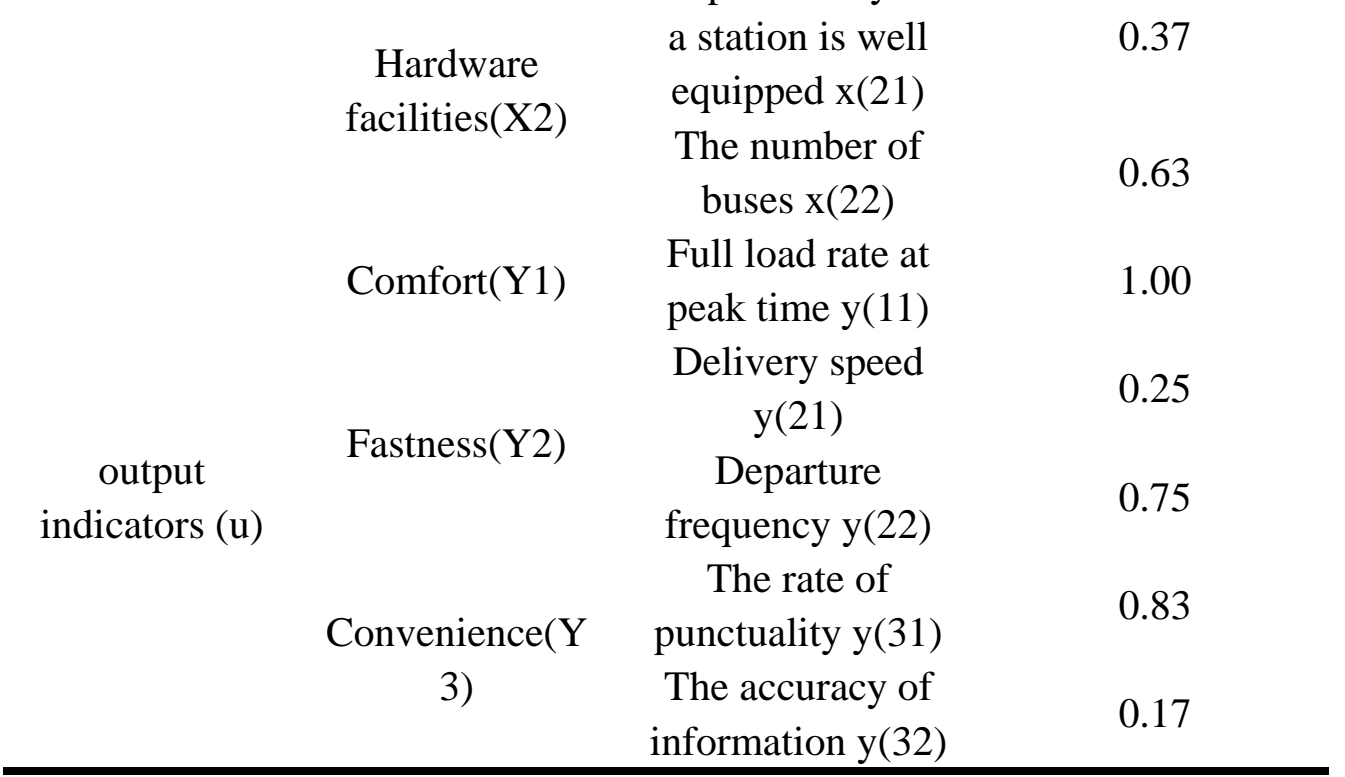

\subsection{The basic principle of DEA method}

Suppose that there are $\mathbf{n}$ decision units DMU, and each decision unit has $\mathbf{m}$ kinds of input indicators and $\mathbf{s}$ kinds of output indicators $x_{i, j}$ indicates that the $\mathrm{j}$-th DMU which corresponds to the value of the i-th input index, $x_{i, j}>0 ; y_{i, j}$ indicates the value of the $\mathrm{j}$-th DMU corresponding to the r-th output index, $y_{i, j}>0$ 。 $v_{i}$ is the weight vector for the i-th input index, $u_{r}$ is the weight vector of the r-th output index, $\mathrm{i}=1, \cdots, m ; j=1, \cdots, s$ 。

Corresponds to a set of weight coefficients:

$$
v=\left(v_{1}, \bullet \bullet \bullet, v m\right)^{T}, u=\left(u_{1}, \bullet \bullet \bullet, u_{s}\right)^{T}
$$

Each decision unit DMU has a corresponding efficiency evaluation index:

$$
h_{j}=\frac{u^{T} y_{j}}{v^{T} x_{j}}=\frac{\sum_{r=1}^{s} u_{r} y_{i j}}{\sum_{i=1}^{m} v_{i} x_{i j}} \cdots \cdots j=1, \cdots, n
$$

Select weight factors $\mathrm{u} 、 \mathrm{v}$ appropriately, make it meet: $h_{j} \leq 1, j=1, \cdots, n$ 。

It can be seen from the above formula, to get the desired integrated efficiency value, the input indicator should be extremely small and the output indicator should 
be extremely large, so the corresponding type of indicators should be transformed accordingly.

To evaluate the efficiency of the $j_{0}$-th decision unit DMU, we consider the weight coefficient $\mathrm{u}, \mathrm{v}$ as variable, make the $j_{0}-$ th decision indicator as target,and consider the efficiency indexes of all decision units as constraints, then build the following optimization model (vector form):

$$
(p)=\left\{\begin{array}{c}
\max \frac{u^{T} y_{j 0}}{v^{T} x_{j 0}} \\
\text { s.t. } \frac{u^{T} y_{j}}{v^{T} x_{j}} \leq 1, j=1,2, \cdots, n \\
v=\left(v_{1}, v_{2}, \cdots, v_{m}\right)^{T} \geq 0 \\
u=\left(u_{1}, u_{2}, \cdots, u_{m}\right)^{T} \geq 0
\end{array}\right.
$$

The optimal solution can be obtained from the model, and the maximum efficiency index can be obtained. The higher the efficiency index is, the better the decision unit DMU is.

The optimization model is a fractional programming problem,using the Charnes-Cooper conversion, it can be transformed into an equivalent linear programming problem.

Make: $t=1 / v^{T} x_{j 0} ; \omega=t \bullet v ; \mu=t \bullet u$, p will transformed into an equivalent linear programming model $\left(\mathrm{P}_{C^{2} R}\right)$ :

$$
\left(P_{C^{2} R}\right)=\left\{\begin{array}{c}
\max h_{j 0}=\mu^{T} y_{j 0} \\
\text { s.t. } \omega^{T} x_{j}-\mu^{T} y_{j} \geq 0, j=1,2, \cdots, n \\
\omega^{T} x_{j 0}=1 \\
\omega=\left(\omega_{1}, \omega_{2}, \cdots, \omega_{m}\right)^{T} \\
\mu=\left(\mu_{1}, \mu_{2}, \cdots, \mu_{s}\right)^{T}
\end{array}\right.
$$

Introducing slack variables and residual variables, we get the following equation: 


$$
\left(D_{C^{2} R}\right)=\left\{\begin{array}{c}
\min \theta \\
\text { s.t. } \sum_{j=1}^{n} \lambda_{j} x_{j}-s^{-}=\theta x_{j 0} \\
\sum_{j=1}^{n} \lambda_{j} y_{j}-s^{+}=y_{j 0} \\
\lambda_{j} \geq 0, j=1,2, \cdots, n \\
s^{-}=\left(s_{1}^{-}, s_{2}^{-}, \cdots, s_{m}^{-}\right)^{T} \geq 0 \\
s^{+}=\left(s_{1}^{+}, s_{2}^{+}, \cdots, s_{s}^{+}\right)^{T} \geq 0
\end{array}\right.
$$

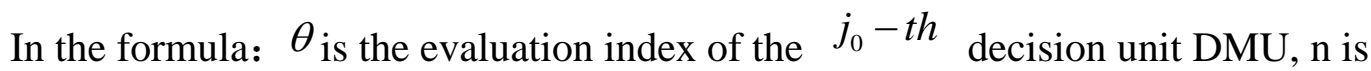
the number of decision units DMU; $\mathrm{S}^{-} 、 S^{+}$, respectively, for the slack variables and residual variables.

2.3 DEA evaluation method based on AHP constraint

The evaluation index system contains more indicators, there is a certain degree of difficulty in the application of DEA method analysiss. Therefore, we need appropriate integration of indicators.To make DEA analysis with comprehensive indicators, the indicators are treated as follows:

$$
Z_{i}^{k}=\sum_{j} \omega_{i j} \sigma_{i j}^{k}
$$

In the formula

$Z_{\mathrm{i}}^{k}$ — The value of the i-th comprehensive index in the k-th decision unit;

$\omega_{\mathrm{ij}}$ — The weight of the $\mathrm{j}$-th sub-indicator in the i-th comprehensive indicator;

$\sigma_{i j}^{k}$ — The value of the $\mathrm{j}$-th sub - index of the $\mathrm{k}$ - th decision unit about the $\mathrm{i}$-th comprehensive indicator.

Using the AHP method, after meeting the consistency test, the relative weight of each index is obtained, and calculate the comprehensive index of each decision unit.

\section{Conclusion}

In this paper,we use DEA evaluation method based on AHP constraint to evaluate the bus line. DEA evaluation method is simple, easy to use. It is used to evaluate decision units with multiple input and output indicators, which is different from the previous assessment method for dealing with individual outputs. The AHP: through a person's subjective intent to construct a judgment matrix,comparing multiple influencing factors, it embodies the basic characteristics of human decision-making thinking.For the model we have built,the optimization and resource allocation of the bus line were defined from a number of aspects and we cmbine AHP with DEA. This model is equally applicable for some of the similar problems in other areas. The scope of the 
model is very broad,for example, in the choice of travel attractions and other common problems.

\section{References}

[1] Bo LIU, Honghong YU. Quantitative bus-line evaluation system based on reliability

[J] .Chinese Journal of Transportation Science and Engineering, 2012, (03): 90-94.

[2] Rongqing HAN. Analysis of Bus Route Utilization in Jinan City Based on MapInfo [J]. Journal of Shandong Normal University (Natural Science Edition), 2008, (03): 114-117.

[3] Meng Qian.Journal of Jilin University (Information Science Edition), 2008, (02): 180-185 (in Chinese with English abstract)

[4]Xiaobo Yang. Study on Evaluation Indexes of Urban Public Traffic Line Network [J]. Science and Technology Innovation Bulletin, 2011, (24): 97-98.

[5] Liheng Shi. Based on MapX bus station layout and line simulation evaluation [D]. Northeastern University, 2010. 